\title{
Factorial invariance of the WHOQOL-BREF among disease groups
}

\author{
Grace Yao \& Chia-huei Wu \\ Department of Psychology, National Taiwan University, Taipei, Taiwan (E-mail: kaiping@ntu.edu.tw)
}

Accepted in revised form 10 March 2005

\begin{abstract}
To compare health-related quality of life (QOL) across groups, researchers have to assure that items of a QOL measure represent the same constructs across groups. This study investigated factorial invariance of the WHOQOL-BREF between a healthy population and populations with diseases using the data from the 2001 National Health Interview Survey (NHIS) in Taiwan. The NHIS was conducted by stratified multistage systematic sampling resulting in responses from 13,010 participants aged 20 65 who completed the WHOQOL-BREF. The analysis was limited to 5 diseases where at least 200 individuals had that specific single condition; these were pulmonary disease, hypertension, peptic ulcer, sinusitis and liver disease. In this study, confirmatory factor analyses (CFA) on a four-factor model were conducted. Two-group analyses with unconstrained/constrained parameters were conducted to confirm the comparability of CFA factor structures between groups. Generally, the $\chi^{2}$ discrepancy tests showed that different disease groups and their matched healthy groups shared the same first- and second-order factor loadings. Moreover, different disease groups shared the same first- and second-order factor loadings as well. In other words, after controlling age and gender, same perceptions on the WHOQOL-BREF questionnaire were found between disease and matched healthy groups and across disease groups.
\end{abstract}

Key words: Confirmatory factor analysis, Factor invariance, Quality of life

\section{Introduction}

In the last 20 years health care providers and researchers have reached a consensus that the efficacy of treatment intervention should be evaluated by its impact on the quantity and the healthrelated quality of life (QOL) [1]. This conception has motivated health care researchers to search for better QOL measures to assess treatment and evaluate cost-effectiveness. In past years, a number of QOL measures have been published, such as the Sickness Impact Profile (SIP), Nottingham Health Profile (NHP), Short-Form-36 Health Survey (MOS SF-36), and recently the World Health Organization QOL questionnaire (WHOQOL). These measurement instruments have been translated into different languages for cross-cultural or cross-national administration. As a result, the issue of cross-cultural or cross-national validation and equivalence of QOL measurement has become an important part of measurement development. For example, it was emphasized at the 2004 annual conference of the International Society for Quality of Life Research (ISOQOL). In addition to conceptual discussion, several empirical methods for measurement equivalence analysis on cross-cultural comparison were proposed at this conference such as structural equation modeling (SEM) and item response theory (IRT).

Although cross-cultural validation and the equivalence of QOL measurement are important for understanding the nature of QOL in different cultures or nations, however, the issue of crossdisease comparison is important as well. The reason is that cross-disease comparison, a common approach for researchers and practitioners on the evaluation of the effectiveness of health care and health resource management, has a direct effect on 
treatment evaluation and resource allocation. In fact, many generic QOL measures that emphasize usability with different populations have been applied to various disease groups and have even been used to compare QOL discrepancies among different disease groups [2-7]. If the constructs underlying QOL measurement are not the same across different disease groups, interpretation of the mean differences in QOL may be problematic. Therefore, researchers should assure that items of a QOL measure represent the same constructs across groups before making group comparisons. In psychometrics, this property has been termed factor invariance.

However, how can the property of factorial invariance for a QOL measurement be examined? Traditionally, QOL researchers have conducted confirmatory factor analysis (CFA) to investigate the factor structure of a QOL measure to confirm whether the observed data represent the same latent structure as previous studies or as other groups. However, they treated groups separately and examined factor structure individually. In other words, researchers may compare the latent structures of various groups through descriptive approaches, leading them to conclude that group A has a similar factor structure to group B just by eyeball examination. Consequently, factor invariance across groups may not be correctly detected.

A common and useful method developed about 30 years ago for examining factor invariance is multiple-group CFA with constrained parameters [8]. Using this method, researchers can test assumptions of factor invariant properties, such as factor structure form, factor loadings, and factor uniqueness variances across several groups under a hypothetical model. This method has been widely used in studies in which factor invariance is examined across groups such as for cross-cultural studies, gender comparison studies, ethic comparison studies, and cross-national studies [9].

The purpose of a QOL study is not only to understand individuals' QOL/health but also to compare QOL across groups (i.e., different genders, diseases, etc.). To achieve this goal, factor invariance across groups is required before meaningful comparisons can be made. To date, very few quantitative studies have compared QOL factor structures across diverse disease groups in healthrelated QOL research. This paper attempts to demonstrate that CFA allows for more meaningful cross-disease comparisons. Applying the correct procedure will result in better understanding of the QOL latent structures for patients with different diseases, and should result in better health care and better health policies with more appropriate treatment and support for patients. Specifically, first- and second-order factor loading invariance of the WHOQOL-BREF questionnaire between the disease-healthy pair groups (i.e., pulmonary disease, hypertension, peptic ulcer, sinusitis, and liver disease) was investigated to demonstrate how factorial invariance of a measurement can be examined.

\section{Method}

\section{Measures}

The WHOQOL-BREF questionnaire was used in this study. The standard WHOQOL-BREF contains 24 facets organized into 4 domains: physical, psychological, social relationships, and environment. The WHOQOL-BREF contains 26 items of which 1 item measures each of the 24 facets and 2 items measure overall QOL and health [10, 11]. Recently, this questionnaire has been culturally adapted to Taiwanese $[12,13]$. In Yao et al.'s study [14], exploratory and confirmatory factor analyses of the WHOQOL-BREF revealed a fourfactor model (physical, psychological, social and environmental factors). The internal consistency (Cronbach's $\alpha$ ) coefficients ranged from 0.70 to 0.77 for the four domains $(0.73-0.83$ in this study using the NHIS sample).

The test-retest reliability coefficients with an interval of 2-4 weeks ranged from 0.41 to 0.79 at the item/facet level and $0.76-0.80$ at the domain level (all $p<0.01$ ). Content validity coefficients ranged from 0.53 to 0.78 for item-domain correlations and from 0.51 to 0.64 for inter-domain correlations (all $p<0.01$ ). All findings revealed that the WHOQOL-BREF has good psychometric properties in Taiwanese as well as in other cultures. To simplify our study, only 24 items from the BREF version were selected; 2 items covering general QOL and health information were excluded as the natures of the 2 items were cross domains that were not appropriate for factor analysis. 


\section{Participants}

The study used data from the 2001 National Health Interview Survey (NHIS) conducted by the National Health Research Institute and the Bureau of Health Promotion (BHP), Department of Health, Taiwan [15]. The 2001 NHIS was intended to provide nation-wide estimates on health conditions, health behaviors, and usage of medical resources for the population in Taiwan. The survey consists of five different questionnaires: a household survey, personal questionnaires (both for those under 12 and those age 12 and over), a youth health behavior questionnaire (for ages 1219), and the WHOQOL-BREF (for ages 20-65, as an optional questionnaire in the NHIS) [16]. On January 16th, 2001, 27,160 eligible persons living in 7357 households were identified through multistage sampling proportional to the size of the household population. The sample was representative of the national population in age, gender, and in terms of the urbanization index [17]. From late August 2001 to January 2002, the 2001 NHIS data were collected from 25,464 persons living in 6721 households, with a response rate of $93.8 \%$ by person and $91.4 \%$ by household [18]. The WHOQOL-BREF questionnaire was administered to 15,425 persons aged 20-65.

Interviewees basically self-administered the questionnaire according to the original design [12$14,19]$. To ensure consistency of the interviews, all interviewers received prior training in which there was a 1 hour item-by-item explanation of the WHOQOL-BREF questionnaire in Taiwanese. Thirteen senior BHP staff closely supervised the interviews, reviewed all completed questionnaires, and randomly verified interviewees' responses through telephone follow-up. They made a crossitem comparison as well between the scale and other corresponding items in the NHIS. No WHOQOL-BREF questionnaires were sent back with an interviewer for a second interview even if there were missing, incorrect, or contradictory responses. No proxy was allowed, even if an interviewee was frail, mentally ill, or unable to communicate. A total of 13,010 out of 15,425 $(84.34 \%)$ persons completed the WHOQOLBREF questionnaire.

From this database, the healthy population was defined as participants without 12 major diseases (i.e., cardiac disease, pulmonary disease, hypertension, diabetic mellitus, hyperlipidaemia, stroke, peptic ulcer, sinusitis, liver disease, renal disease, prostate disease, and uterine/ovary disease), blindness and cancers as diagnosed by a doctor. As a result, 9109 participants were in the Health group. In addition, several disease groups were established. In order to examine factor invariance across the different disease groups and attribute the results to those diseases, participants with multiple major diseases were excluded from specific disease groups. In other words, participants in each group had only one major disease. Moreover, to make data analysis more meaningful, only disease groups with a substantial number of participants (approximate to 200 or more) were retained for further analysis. Accordingly, populations of the pulmonary disease $(\mathrm{n}=235)$, hypertension $(\mathrm{n}=487)$, peptic ulcer $(\mathrm{n}=487), \quad$ sinusitis $(\mathrm{n}=376)$, and liver disease $(\mathrm{n}=500)$ groups were used for further analyses.

Table 1 presents the demographic data for participants from the healthy and disease populations. The first column in Table 1 shows the demographic data for the healthy population and the five disease populations before exclusion of participants with multiple diseases. The second column in Table 1 shows the demographic data for each disease group for participants with only a single disease. Finally, the third column in Table 1 shows the demographic data for a matched healthy group for each disease group. These matched healthy groups were selected from the healthy population to make a reasonable comparison between disease and healthy population. Matched healthy groups were necessary as the demographic variables of the participants in the disease groups were different from those of the healthy population. If participants' demographic variables were not controlled, the results of factor invariance analysis would not necessarily be attributable to the disease dimension. Moreover, the size of the healthy population $(n=9109)$ was much larger than the size of each individual disease group. Consequently, parameter estimates would have been distorted in multiple-group CFA analysis if the healthy group was used for comparison. As Bollen indicated [20], in multiple-group CFA analysis the larger sample receives greater weight in the minimizing general fit function so parameter 


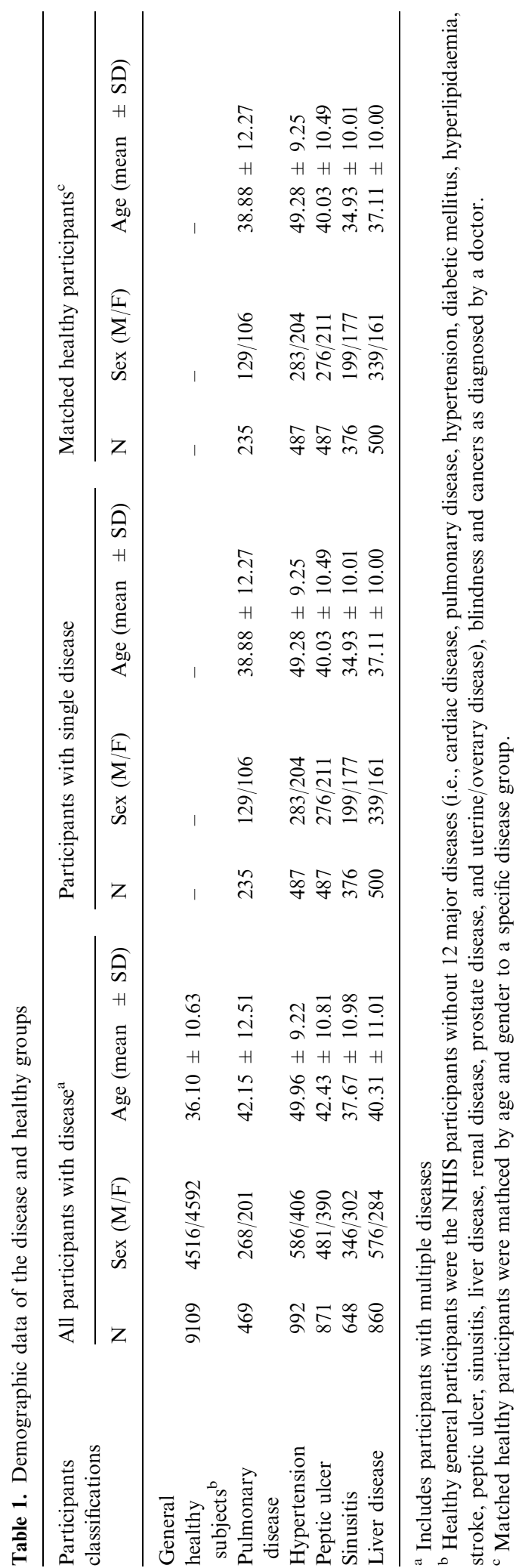

estimates tend toward the larger sample. To avoid these problems and make reasonable comparisons among groups, a matched healthy population was selected from the healthy population for each individual disease group. Specifically, participants in each matched healthy group were identified by matching their age and gender with the participants in a particular disease group. These two demographic variables showed a significant relation with WHOQOL scores in a preliminary analysis [21]. If a number of healthy participants matched the criteria, random selection was used to select the required number of participants to form a matched healthy group.

\section{Analytical methods}

According to the studies on the WHOQOL-BREF [11, 14, 19], a four-factor model was appropriate for the WHOQOL-BREF questionnaire, so a fourfactor model for CFA was specified. For CFA, multi-sample analyses were conducted using LISREL software [22]. Multi-sample analyses can examine several models simultaneously with some or all parameters constrained to be equal over groups $[22,23]$. Specifically, in this study only the parameters of the pre-specified four-factor model were constrained with two factor levels for two groups in a hierarchical order. The parameters for the first-order factor level refer to the factor loadings between domains (factors) and their indicators (variables). The parameters for the second-order factor level refer to the factor loadings between the general QOL factor and domain factors. As a result, three hierarchical models were proposed in order to compare the differences between the two groups. Model A does not specify any constraint on the two groups. Model B specifies all the first-order factor loadings as equal on the two groups. Model $\mathrm{C}$ specifies the entire firstand the second-order factor loadings as equal on the two groups. The $\chi^{2}$ values of Models A, B and $\mathrm{C}$ are summarized for all pair group comparisons under examination. With consideration of the corresponding $\mathrm{df}$, the $\chi^{2}$ differences between Model A and Model B and between Model B and Model $\mathrm{C}$ allow the possibility of choosing the model with more constraints. For example, the $\chi^{2}$ value and df of Model A for comparing the pulmonary disease group and its matched healthy 
Table 2. The $\chi^{2}$ value (with $\mathrm{df}$ ) for models $\mathrm{A}, \mathrm{B}$, and $\mathrm{C}$ on pairs of disease and matched healthy groups

\begin{tabular}{lll}
\hline & $\begin{array}{l}\text { N (disease } \\
\text { group/healthy } \\
\text { group) }\end{array}$ & $\chi^{2}(\mathrm{df})$ \\
\hline Pulmonary disease & $235 / 235$ & A: $\chi^{2}(496)=1271.61$ \\
& & B: $\chi^{2}(516)=1300.12$ \\
Hypertension & C: $\chi^{2}(520)=1307.67$ \\
& A: $\chi^{2}(496)=1782.19$ \\
Peptic ulcer & B: $\chi^{2}(516)=1814.70$ \\
& C: $\chi^{2}(520)=1820.68$ \\
Sinusitis & A: $\chi^{2}(496)=1816.19$ \\
& B: $\chi^{2}(516)=1830.11$ \\
Liver disease & C: $\chi^{2}(520)=1835.99$ \\
& A: $\chi^{2}(496)=1474.92$ \\
& B: $\chi^{2}(516)=1491.05$ \\
& C: $\chi^{2}(520)=1495.63$ \\
& A: $\chi^{2}(496)=1816.60$ \\
& B: $\chi^{2}(516)=1839.13$ \\
& C: $\chi^{2}(520)=1840.02$ \\
\hline
\end{tabular}

Note: ${ }^{*} \chi^{2}$ difference test was significant between Models A and B. Critical values: $\chi^{2}(\alpha=0.01, \mathrm{df}=20)=37.57 ; \chi^{2}(\alpha=0.01$, $\mathrm{df}=4)=13.28$.

group pair was 1271.61 and 496 respectively, and 1300.12 and 516 for Model B (see Table 2). The discrepancy of the $\chi^{2}$ values between Model A and Model B was $1300.12-1271.61=28.51$ with $\mathrm{df}=516-496=20$. This $\chi^{2}$ discrepancy was then tested to see whether it was statistically significant with its corresponding df. If the result is statistically significant, the model with less constraint is the better model and it may be concluded that the QOL of the participants in the two groups have different factor loadings. In this example, the $\chi^{2}$ discrepancy test was not significant $\left(\chi^{2}\right.$ $(20)=28.51, p>0.05$, with a critical value of 37.57), suggesting that Model $\mathrm{B}$ was better than Model A because of its parsimony. This indicates that the first-order factor loadings of the pulmonary disease group and its matched healthy group were not different. If the $\chi^{2}$ difference test between Model A and Model B is not significant (i.e., the two groups have the same perceptions on variables in its first-order factor), then, a $\chi^{2}$ difference test between Model B and Model C is conducted. However, if the $\chi^{2}$ difference test between Model A and Model B is significant (i.e., the two groups have different perceptions on variables in its firstorder factor), then, a $\chi^{2}$ difference test between
Model B and Model C is meaningless and should not be conducted.

In addition, in considering the number of comparisons that were made in the following analysis, the probability of Type I errors were controlled by Bonferroni adjustment method. Since first- and second-factor loadings were regarded as different part, $\chi^{2}$ difference tests on Model A-Model B (testing invariance on first-factor loadings) and Model B-Model C (testing invariance on secondfactor loadings) were treated as two families of contrasts. Therefore, the $\alpha$ level of $\chi^{2}$ difference test of the comparisons between each healthydisease pair was $0.01(0.05 / 5)$ for both the Model A-Model B and Model B-Model C contrasts. Similarly, the $\alpha$ level of $\chi^{2}$ difference test of the comparisons between each of the 10 disease-disease pairs was $0.005(0.05 / 10)$ for both the Model A-Model B and Model B-Model C contrasts.

\section{Results}

The $\chi^{2}$ value (with $\mathrm{df}$ ) for model comparison was reported in Table 2 for each pair-group. According to the results, the fit indices of Model A for the pair of each disease group and its matched healthy group suggested that the four-factor model was acceptable $(\mathrm{CFI}=0.94 \sim 0.96$; $\mathrm{RMSEA}=0.075 \sim 0.083$; $\mathrm{TLI}=0.94 \sim 0.95$ ). The four-factor model was a reasonable model for disease groups and their matched healthy groups.

Subsequently, Model B was fit for each pairgroup. According to the $\chi^{2}$ discrepancy tests between Models A and B, all contrasts were not significant, suggesting that the first-order loadings (between domains and indicators/items) of each disease group were the same as those of matched healthy population. In addition, $\chi^{2}$ discrepancy tests between Model B and C for disease-healthy group pairs were also insignificant (see Table 2). This result suggests that Model $\mathrm{C}$ was better than Model A and B for each disease-healthy group pair. In other words, each disease group and its matched healthy group had the same first- and second-order factor loadings.

In summary, patients and their matched healthy populations have the same four-factor QOL structure; moreover, the first- and the 
Table 3. The $\chi^{2}$ value (with $\mathrm{df}$ ) for models $\mathrm{A}, \mathrm{B}$, and $\mathrm{C}$ on pairs of disease groups

\begin{tabular}{|c|c|c|c|c|c|}
\hline & $\begin{array}{l}\text { Pulmonary } \\
\text { disease } \\
(\mathrm{n}=235)\end{array}$ & $\begin{array}{l}\text { Hypertension } \\
(\mathrm{n}=487)\end{array}$ & $\begin{array}{l}\text { Peptic ulcer } \\
(\mathrm{n}=487)\end{array}$ & $\begin{array}{l}\text { Sinusitis } \\
(\mathrm{n}=376)\end{array}$ & $\begin{array}{l}\text { Liver disease } \\
(\mathrm{n}=500)\end{array}$ \\
\hline \multicolumn{2}{|c|}{ Pulmonary disease } & $\begin{array}{l}\mathrm{A}: \chi^{2}(496)=1564.90 \\
\mathrm{~B}: \chi^{2}(516)=1579.02 \\
\mathrm{C}: \chi^{2}(520)=1584.74\end{array}$ & $\begin{array}{l}\text { A: } \chi^{2}(496)=1571.42 \\
\mathrm{~B}: \chi^{2}(516)=1600.32 \\
\mathrm{C}: \chi^{2}(520)=1605.18\end{array}$ & $\begin{array}{l}\text { A: } \chi^{2}(496)=1416.91 \\
\text { B: } \chi^{2}(516)=1445.83 \\
\text { C: } \chi^{2}(520)=1449.22\end{array}$ & $\begin{array}{l}\text { A: } \chi^{2}(496)=1513.49 \\
\text { B: } \chi^{2}(516)=1539.98 \\
\text { C: } \chi^{2}(520)=1549.70\end{array}$ \\
\hline \multicolumn{2}{|l|}{ Hypertension } & & $\begin{array}{l}\text { A: } \chi^{2}(496)=1853.58 \\
\text { B: } \chi^{2}(516)=1880.15 \\
\text { C: } \chi^{2}(520)=1884.02\end{array}$ & $\begin{array}{l}\text { A: } \chi^{2}(496)=1699.07 \\
\text { B: } \chi^{2}(516)=1735.60 \\
\text { C: } \chi^{2}(520)=1737.09\end{array}$ & $\begin{array}{l}\text { A: } \chi^{2}(496)=1795.65 \\
\text { B: } \chi^{2}(516)=1818.47 \\
\text { C: } \chi^{2}(520)=1820.41\end{array}$ \\
\hline \multicolumn{2}{|l|}{ Peptic ulcer } & & & $\begin{array}{l}\text { A: } \chi^{2}(496)=1705.59 \\
\text { B: } \chi^{2}(516)=1729.97 \\
\text { C: } \chi^{2}(520)=1732.13\end{array}$ & $\begin{array}{l}\mathrm{A}: \chi^{2}(496)=1802.17 \\
\mathrm{~B}: \chi^{2}(516)=1826.53 \\
\mathrm{C}: \chi^{2}(520)=1831.63\end{array}$ \\
\hline Sinusitis & & & & & $\begin{array}{l}\text { A: } \chi^{2}(496)=1647.66 \\
\text { B: } \chi^{2}(516)=1668.91 \\
\text { C: } \chi^{2}(520)=1671.67\end{array}$ \\
\hline
\end{tabular}

Note: $* \chi^{2}$ difference test was significant between Models B and C.

Critical values: $\chi^{2}(\alpha=0.005, \mathrm{df}=20)=39.99 ; \chi^{2}(\alpha=0.005, \mathrm{df}=4)=14.86$.

second-order factor loadings were all equal. This indicates that patients and their matched healthy populations may have the same interpretations on the items of the WHOQOL questionnaire.

Additionally, each pair of the five disease groups (pulmonary disease, hypertension, peptic ulcer, sinusitis, and liver disease) was also compared. Table 3 shows that $\chi^{2}$ discrepancy tests between Model A and Model B were not significant, which suggests that all disease pairs had the same firstorder factor loadings. In addition, the $\chi^{2}$ discrepancy tests between Model B and Model C also had non-significant differences for all disease pairs, showing that all disease pairs had the same secondorder factor loadings (between domains and general QOL). As a result, it is acceptable to say that participants in each of the disease groups in this study had the same interpretations on the WHOQOL questionnaire items.

\section{Discussion and conclusions}

This paper has shown that QOL factor structures constructed by CFA can be compared between healthy and disease groups using multiple-group approach. By model comparison, it is a desirable method to examine the factor invariance properties of a well-established measurement [9]. First, the multiple-group CFA approach simultaneously provides a factor solution and the best estimates across groups. In addition, it provides a strong hypothetical test of whether invariance factors have (or have not) been obtained. In other words, multiple-group CFA provides strict information on the equality of estimates across groups.

Under this theoretical background, the factor invariance of the WHOQOL-BREF questionnaire across different disease and healthy groups were examined in this study. According to the model comparison results, the $\chi^{2}$ discrepancy tests showed that different disease groups and their matched healthy groups shared the same first- and second-order factor loadings. Moreover, different disease groups shared the same first- and secondorder factor loadings as well. In other words, after controlling age and gender, the disease groups had the same perceptions on the WHOQOL questionnaire as other disease groups and the healthy population. While no evidence of non-invariance was found in the current study, we would like to mention that if it were observed, further studies still need to be done. This is because the CFA results on factor invariance can only illustrate the phenomenon, but do not explain how and why these non-invariant results occur. Therefore, the examination of factor invariance is the initial step for investigating the equality of scale constructs 
across groups. If a non-invariant result is found, further investigation should be conducted to examine the nature of the differences underlying the constructs between the analyzed groups.

In conclusion, this study has compared factor structures between disease and matched healthy groups and among disease groups. The results showed that quantitative comparisons on latent structures are possible across different healthy and disease groups. However, there is a major limitation to this study. That is, in order to clearly attribute the results of factor invariance analysis to a specific disease, participants with only a single major disease were selected. The fact that people with one disease may also have other diseases was ignored. For example, people with cardiac disease often have hypertension as well. As a result, the single disease comparison approach does not reflect the true phenomenon of multiple diseases. However, considering the purpose of factor invariance analysis across disease groups and the assumptions of the multiple-group CFA approaches, this single disease comparison approach is acceptable. To study factor invariance with multiple-group CFA, independent samples are definitely required. If a disease group was not formed by selecting participants with only one major disease, the participants for two compared groups might have overlapped, and the results would be contaminated and not attributable to the difference in the two groups. Therefore, although the single disease comparison approach may have some limitations, it is still a reasonable and acceptable method. Another limitation of this study is that population-based participants were used in this study. As a result, participants with severe physical conditions requiring hospitalization were not included.

Finally, age differences among disease groups should also be mentioned. Among the five disease groups, the oldest was the hypertension group with mean age of 49.28, and the youngest was the sinusitis group with mean age of 34.93. Although there are age differences among these groups, according to the current findings, all groups have the same first- and second- factor loadings. This finding might show that the age differences would not lead to different factor structure underlying the WHOQOL-BREF. However, among all compari- sons, the comparison of sinusitis vs. hypertension was close to statistical significance (the $p$ value of Model A-Model B contrast was 0.01 with the criterion of 0.005), which might result from the age difference between the two groups. Accordingly, the role of age difference on the factor invariance of the WHOQOL-BREF should be examined in the future.

Generally, in future studies researchers can use the same approach to examine whether factor invariance on the same measurement exists across different groups (by genders, diseases, cultures, etc.). Group comparisons are meaningful only when factor invariance exists across groups.

\section{Acknowledgements}

This study was supported by the National Science Council (NSC89-2519-S-002-003-, NSC90-2511-S002-009-, NSC91-2519-S-002-002-, NSC 92-2320B-002-172 NHRI-EX94-9204PP). We thank Dr. Diane L. Fairclough for her invaluable suggestions and help on the early draft of this paper. We also acknowledge the National Health Research Institute and the Bureau of Health Promotion (BHP), Department of Health, Taiwan for providing the NHIS data.

\section{References}

1. Leung KF, Tay M, Cheng SW, et al: Hong Kong Chinese version World Health Organization quality of life measure - abbreviated version. (WHOQOL-BREF (HK)), 1997.

2. Alonso J, Ferrer M, Gandek B, et al. Health-related quality of life associated with chronic conditions in eight countries: Results from the International Quality of Life Assessment (IQOLA) Project. Qual Life Res 2004; 13: 283-298.

3. Arnold R, Ranchor AV, Sanderman R, et al. The relative contribution of domains of quality of life to overall quality of life for different chronic diseases. Qual Life Res 2004; 13: 883-896.

4. Lam CLK, Lauder IJ. The impact of chronic diseases on the health-related quality of life (HRQOL) of Chinese patients in primary care. Fam Prac 2000; 17: 159-166.

5. Lam CLK, Lauder IJ, Lam TPD. The impact of chronic diseases on health services and quality of life of a Chinese population. Asia Pac Fam Med 2003; 2: 98-106.

6. Patrick DL, Kinne S, Engelberg RA, Pearlman RA. Functional status and perceived quality of life in adults with and without chronic conditions. J Clin Epidemiol 2000; 53: 779-785. 
7. Sprangers MAG, de Regt EB, Andries F, et al. Which chronic conditions are associated with better or poorer quality of life? J Clin Epidemiol 2000; 53: 895-907.

8. Joreskog KG. Simultaneous factor analysis in several populations. Psychometrika 1971; 57: 409-426.

9. Vandenberg RJ, Lance CE. A review and synthesis of the measurement invariance literature: Suggestions, practices and recommendation for organizational research. Organ Res Methods 2000; 3: 4-70.

10. The WHOQOL Group: The World Health Organization Quality of Life assessment (WHOQOL): Development and general psychometric properties. Soc Sci Med 1998; 46: $1569-1585$.

11. The WHOQOL Group. Development of the World Health Organization WHOQOL-BREF quality of life assessment. Psychol Med 1998; 28: 551-558.

12. The WHOQOL-Taiwan Group. Introduction to the development of the WHOQOL-Taiwan version. Chinese J Public Health (Taipei) 2000; 19: 315-324.

13. The WHOQOL-Taiwan Group: The User's Manual of the Development of the WHOQOL-BREF Taiwan Version. 1st rev. ed. Taiwan: Taipei, 2001.

14. Yao G, Chung CW, Yu CF, Wang JD. Development and verification of validity and reliability of the WHOQOLBREF Taiwan version. J Formos Med Assoc 2002; 101: 342-51.

15. Shih YT, Chang HY, Le KH, et al. Introduction to the National Health Interview Survey (NHIS). NHIS Brief Communication No. 1, June 2002.
16. Chang FC, Chang HY, Liu HY, Wu SL. Introduction to the National Health Interview Survey (NHIS) Questionnaire design. NHIS Brief Communication No. 3, June 2002.

17. Hung YT. Sampling design of the National Health Interview Survey. NHIS Brief Communication No. 2, June 2002.

18. Lin SH. Field collection and completeness of data in the National Health Interview Survey. NHIS Brief Communication No. 4, June 2002.

19. World Health Organization. WHOQOL-BREF: Introduction, administration, scoring and generic version of the assessment - Field trial version. Geneva: WHO, 1996.

20. Bollen KA. Structural equations with latent variables. New York: John Wiley, 1989.

21. Yao G, Wang JD, Chen CM, Tsai, YJ. Cross-validation and normative analysis of establishment of the WHOQOLBREF Taiwan version. Paper presented at the 10th Annual Conference of the International Society for Quality of Life Research, Czech Republic, 2003.

22. Joreskog KG, Sorbom D. LISREL 8: Structural Equation Modeling With the SIMPLIS Command Language. Hillsdale, NJ: Lawrence Erlbaum Associates, 1993.

23. Bentler PM. EQS Structural Equations Program Manual. Los Angeles, CA: BMDP Statistical Software, 1993.

Address for correspondence: Grace Yao, Department of Psychology, National Taiwan University 1, Sec. 4, Roosevelt RD., Taipei, Taiwan

Phone: 886-2-3366-3098; Fax: 886-2-2362-9909

E-mail: kaiping@ntu.edu.tw 